\title{
Comunidade de aves da Reserva Estadual de Gurjaú, Pernambuco, Brasil
}

\author{
Rachel Maria de Lyra-Neves 1, Manoel Martins Dias 2, Severino Mendes de Azevedo-Júnior 1,3, \\ Wallace Rodrigues Telino-Júnior ${ }^{3,4}$ \& Maria Eduarda Lacerda de Larrazábal ${ }^{3}$
}

${ }^{1}$ Departamento de Biologia, Área de Zoologia, Universidade Federal Rural de Pernambuco. Avenida Dom Manuel de Medeiros, Dois Irmãos, 52171-900 Recife, Pernambuco,Brasil.E-mail: rmlneves@msn.com

2 Departamento de Ecologia e Biologia Evolutiva, Universidade Federal de São Carlos. Caixa Postal 676, 13565-905 São Carlos, São Paulo, Brasil.

${ }_{3}^{3}$ Departamento de Zoologia, Centro de Ciências Biológicas, Universidade Federal de Pernambuco. Avenida Professor Moraes Rego 1235, Cidade Universitária, 50670-420 Recife, Pernambuco, Brasil.E-mail: smaj@ufpe.br

${ }_{4}^{4}$ IBAMA/CEMAVE. Rodovia BR 230, Km 10, Mata da Amem, 58300-000 Cabedelo, Paraíba, Brasil. E-mail: telinojr@msn.com

\begin{abstract}
Bird Communities of the Gurjaú Reserve, Pernambuco State, Brazil. A qualitative and quantitative bird surveys were carried out in a forest fragment in Gurjau Reserve, Santo Agostinho Cape, Pernambuco State, Brazil. By qualitative census were registered 220 different bird species. Concerning the point counts, were identified 175 different species in 6.470 contacts (270 samples). A frequency of occurrence of $75 \%$ was registered for 43 species $(19,6 \%)$; the most part of species had a frequency of occurrence below $25 \%$.

KEY WORDS. Atlantic Rain Forest, Neotropical birds, point counts.
\end{abstract}

RESUMO. Estudos quali-quantitativos foram realizados em um fragmento florestal da Reserva Estadual de Gurjaú, Cabo de Santo Agostinho, Pernambuco, Brasil. Foram registradas 220 espécies de aves no levantamento qualitativo. Através da contagem por pontos, foram identificadas 175 espécies em 6.470 contatos ( 270 amostras). A frequiência de ocorrência de $75 \%$ foi registrada para 43 espécies (19,6\%); para a maioria das espécies a freqüência de ocorrência esteve abaixo de $25 \%$.

PALAVRAS CHAVE. Aves neotropicais, contagem por pontos de escuta, Mata Atlântica.

\begin{abstract}
A Mata Atlântica é considerada o bioma de maior diversidade biológica do planeta, embora restem apenas 10\% de sua cobertura original, sendo uma área crítica devido à pressão e destruição exercida pelo ser humano (WILlis \& ONIKI 1992). Possui alto nível de endemismo em todos os grupos taxonômicos, incluindo aves, primatas, borboletas e plantas (PRIMACK \& Rodrigues 2001). Constitui área chave para espécies de aves ameaçadas nos neotrópicos (Wege \& Long 1995), além de ser considerada um dos oito hot spot mais críticos do mundo, devido ao alto grau de endemismo e mais de $70 \%$ de sua vegetação nativa descaracterizada (Myers et al. 2000, BRоoкs et al. 2002).

O Nordeste do Brasil é o setor mais ameaçado da Mata Atlântica em conseqüência do desmatamento, restando apenas 2\% de sua área original (Silva \& TABARelli 2000). Ranta et al. (1998) ao estudarem áreas na Zona da Mata Sul do Estado de Pernambuco detectaram que $48 \%$ dos fragmentos são menores que 10 ha e somente $7 \%$ maiores que 100 ha. O desmatamento, principalmente para plantio da cana-de-açúcar, exerce grande pressão sobre os fragmentos que ainda restam, comprometendo-os de forma irreparável.
\end{abstract}

O desmatamento resulta na transformação de amplas áreas de floresta primária em mosaicos de pastagem e fragmentos florestais, tendo sérias conseqüências para a biodiversidade (Bierregaard et al. 1992). Para o estado de Pernambuco a canade-açúcar foi a principal causa do desmatamento florestal no século passado. Esta fragmentação ameaça a existência das espécies e há formação de uma quantidade maior de borda de florestas, por área de hábitat, conhecida como efeito de borda, tendo uma conseqüência bastante negativa, não apenas sobre as aves dependentes de interior de floresta, mas também sobre outras populações (Primack \& RodRigues 2001).

A situação atual das florestas tropicais despertou uma necessidade urgente de sua preservação, devido a enorme biodiversidade e a fragilidade desses ecossitemas (VIELLIARD 2000). Segundo MARINI (2000) os efeitos deletérios da fragmentação florestal sobre as comunidades de aves, não são totalmente conhecidos. De acordo com Vielliard (2000), vários métodos têm sido utilizados para o estudo desses ecossistemas (e. g. BierRegaARD \& Lovejoy 1989, Stouffer \& BierregaARd Jr. 1995a, b, STotz et al. 1996), porém, não fornecem uma imagem global da comunidade estudada. 
A contagem por pontos de escuta, segundoBLondel et al (1970) éum método bastante eficientee defácil implementação nos neotrópicos (Aleixo \& Vielliard 1995), propiciando uma amostragem representativa de hábitats heterogêneos, além de permitir a mel hor detecção de espécies. Por outro lado, a interpretação matemática é mais precisa e fácil de ser calculada (Vielliard \& Silva 1990, Viellard 2000).

Trabal hos com esse enfoquetêm sido desenvolvidos com maior regularidadeno sudesteesul do Brasil, a exemplo deA Lexo (1999), Aleixo \& Vielliard (1995), Almeida et al. (1999), Anjos (1998, 2001, 2002), Anjos \& Boçon (1999), Gimenes \& Anjos (2000), M arsden et al. (2001), Viellard \& Silva (1990), Viellard (2000).

No Nordeste do Brasil não há publicações sobre ecologia quantitativa da avifauna de Mata Atlântica. Neste sentido o principal objetivo destetrabal ho foi o estudo da abundância e diversidade da avifauna em um fragmento florestal da Zona da Mata, sul do Estado de Pernambuco, através da contagem por pontos de escuta.

\section{MATERIAL E MÉTODOS}

\section{Área de estudo}

A Reserva de Gurjaú está localizada nos municípios de Cabo de Santo Agostinho, M oreno eJaboatão dos Guararapes, Zona da Mata, sul do Estado de Pernambuco (814'21,7"S e 35003'00,4"W). Limita-se com a Usina Bom Jesus e os Engenhos Salvador e Espírito. Está inserida em uma única proprie dade pública, pertencente à Companhia Pernambucana de Abastecimento deÁgua (COM PESA), onde existe uma estação de tratamento. No interior da reserva encontram-se três açudes: Sucupema, Gurjaú e São Salvador (FIDEM 1987).

Possui uma área total de $1.077,10$ ha, distribuídos segundo a Fidem (1987), em: 744,47 ha no Cabo de Santo Agostinho; 175,19 ha em M oreno e157,44 ha em Jaboatão dos Guararapes. É definida como Reserva Estadual pela Lei Estadual no 9.989 de 1987. Apesar de ser um remanescente de Mata Atlântica, sua cobertura vegetal não é uniforme, apresentando trechos de vegetação densa e outros bastante al terados em sua estrutura e fisionomia (FIDEM 1987). Essa área encontra-se bastante ameaçada pela exploração canaviei ra euso indiscriminado por poceiros, os quais utilizam a área para agricultura de subsistência, fruticultura e retirada de madeira.

\section{Métodos}

Esta pesquisa teve duração de nove meses, compreendendo as estações seca e chuvosa, com expedições mensais de seis dias cada entre agosto de 2002 e abril de 2003. A amostragem da avifauna foi realizada por análise qualitativa e quantitativa através de contagem por pontos segundoBLONDEL et al. (1970) e Vielliard \& Silva (1990).

O levantamento quantitativo teve início a partir do alvorecer, com término às dez horas, durante três dias consecutivos, totalizando 27 visitas. As contagens foram realizadas em uma trilha pré-existente na Reserva com 10 pontos previamente definidos, distanciados de 400 metros, abrangendo o interior e a borda da mata. As espécies foram registradas por 20 minutos em pontos pré-determinados. Foram an otados: visualizações e/ou vocalizações a cada contato estabel ecido, estrato ocupado, número de indivíduos (exemplares isolados, em pares ou em grupos). Casais e grupos familiares foram considerados como apenas um indivíduo, de acordo com VieLLIARD \& SilVA (1990) e Vielliard (2000).

Para o levantamento qualitativo, além daquelas espécies já registradas no quantitativo, também foram realizadas caminhadas pelo interior eborda dosfragmentosenasáreas adjacentes com pontos de escuta e observação, de acordo com (ALMEIDA et al. 1999), entre os horários das cinco às dez horas da manhã e das três as cinco e meia da tarde, durante cinco dias consecutivos em nove meses, totalizando 337,5 horas de observação.

As vocalizações foram gravadas com o auxílio de minicassete Aiwa TP560 e microfone Leson ML8. As vozes das aves não identificadas no local, foram comparadas com gravações pré-existentes ou por meio de consulta a especial istas.

Calculou-se: 1) freqüência de ocorrência (FO) e 2) índice pontual de abundância (IPA). A FO demonstra a relação entre a proporção dos dias em que a espéciei foi observada dividida pelo número total de dias amostrados (dias $x$ meses), as espécies foram classificadas em residentes, vagantes, ocasionais e migratórias de acordo com ALmEIDA et al. (1999). O IPA repre senta o número total de contatos da espécie i em relação ao número total de amostras (visitas x pontos). Esteval or é rel ativo e segundo (AlEIXo \& VielLIARD 1995) é comparável somente entre medidas da mesma espécie em datas, locais e comunidades diferentes.

A variação encontrada no número de espécies por visita, expressa em percentuais, foi cal culada da seguinte forma: número de espécie por visita ( $>$ no espécie por visita $-<n$ 으 espécie por visita $\div<\mathrm{n}$ o espécie por visita); IPA médio mensal (>IPA médio mensal - <IPA médio mensal $\div<$ IPA médio mensal).

Para avaliar a diversi dade da avifauna de Gurjaú, utilizaram-se osÍndices de Shannon-Wiener e de Eqüitabilidade, aplicados aos registros de abun dância rel ativa das espécies, de acordo com MagURRAN (1988).

\section{RESULTADOS E DISCUSSÃO}

Foram registradas 220 espécies para a Reserva Estadual de Gurjaú, quecorrespondea $44,18 \%$ da avifauna de Pernambuco (498 espécies). Este total é alto se comparado a outros levantamentos real izados em dois fragmentos da Estação Ecológica do Tapacurá por AzEvedo-J únIor (1990) com 181espécies, e também, em seis fragmentos da Área de Proteção Ambiental de Guadalupe, localizada na mata sul de Pernambuco, desenvolvidos por LyRA-NeVEs et al. (2000), com registro de 187 espécies.

Apenas $19,6 \%$ das espécies $(n=43$ ) apresentaram FO acima de $75 \%$, sendo consideradas residentes abundantes, a exemplo de: Leptotila rufaxilla (Richard \& Bernard, 1792); Phaethornis ruber (Linnaeus, 1758); Elaenia flavogaster (Thunberg, 1822); 
Hemitriccus zosterops (Pelzeln, 1868); Todirostrum cinereum (Linnaeus, 1766); Tolmomyiasflaviventris (Wied, 1831); Pitangus sulphuratus (Linnaeus, 1766); Chiroxiphia pareola (Linnaeus, 1766); Thryothorus genibarbis Swainson, 1837; Troglodytes musculus Naumann, 1823; Turdus leucomelas Vieillot, 1818; Cyclarhis gujanensis (Gmelin, 1789); V ireo chivi (Vieillot, 1817); Thraupis palmarum (Wied, 1821) e Saltator maximus (Müller, 1776), todos com FO de $100 \%$ (Tab. I).

O percentual de espécies com $\mathrm{FO}$ acima de $75 \%$, foi maior que o registrado por ALm EIDA et al. (1999), com apenas 7\% de espécies com essa freqüência. Entretanto, se comparados aos resultados obtidos por KRügel \& Anjos (2000) em que 50,9\% da avifauna amostrada obtiveram freqüência de ocorrência acima de $76 \%$, foi baixo.

As espécies com percentual abaixo de $25 \%$ das amostras, foram a grandemaioria, com $46,8 \%$, aparecendo em no máximo cinco das 27 visitas. Conforme resultados de Aleixo \& VielLIARD (1995), a maior parte da avifauna (44\%) era também constituída por espécies com FO inferior a 25\%. KRÜGEL \& ANjos (2000), obtiveram percentuais menores, sendo $23,7 \%$ poucas visistas e 13,1\% em apenas uma.

As espécies Pyrigl ena leuconota (Spix, 1824), Conopophaga melanops (Wied, 1831), Platyrinchus mystaceus Vieillot, 1818 e Myiobius barbatus (Gmelin, 1789), obtiveram o status de vagante, com registro em apenas uma visita. AleIxo \& VIELLIARD (1995) e ALm EIDA et al. (1999), explicam que espécies consideradas vagantes ou ocasionais, com baixa freqüência de ocorrência, podem estar relacionadas à pouca relação com os ambientes da mata, ou seja, espécies que permanecem poucos dias na área e aquel as que habitam outros ambientes e ocasional mente exploram recursos da mata, levando a um registro esporádico; a baixa densidade populacional da espécie na área e espécies com vocalizações pouco conspícuas.

StoufFer \& BierRegAard (1995b) relatam o desaparecimento de espécies insetívoras de subosque, principalmente as seguidoras de correição (e.g. P. leuconota), que são as primeiras a desaparecerem com as alterações ambientais (BIERREGAARD \& Lovejoy 1989). Segundo Askins et al. (1987) e Stouffer \& BierRegaARd J R. (1995b), espécies dependentes de interior de floresta são vulneráveis à fragmentação.

Foram catalogadas 175 espécies (média de $73 \pm 8,5$ ) em 270 amostras ( 10 amostras/27 visitas) com 6.470 contatos, obtendo-se média de 24,0 $\pm 7,6$ contatos/amostra. Estes dados se comparados aos encontrados por outros autoresébastante elevado (Tab. II ). O número de espécies variou entre 45 e 89 por visita (Fig. 1). Os menores números foram registrados nos meses chuvosos e os maiores nos meses de estiagem ou reprodutivo, quando al gumas espécies se tornam mais evidentes devido à emissão de vocal izações que ocorre neste período. A variação foi de $97,8 \%$ sendo inferior àquela encontrada por A Aeixo \& VIELLIARD (1995) com 102\%. Entretanto, se assemel ha aos resultados obtidos por VIELLIARD \& SILVA (1990) com variação de $92 \%$.

A média mensal de espécies por visita variou de $61 \pm 14$

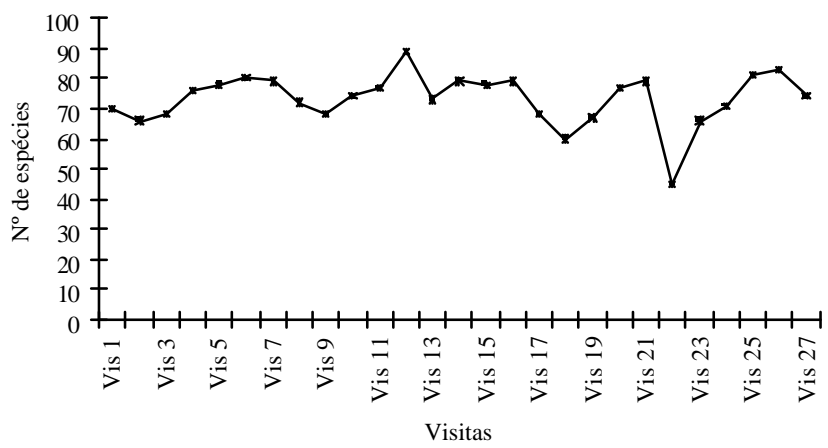

Figura 1. Variação do número de espécies encontradas por visita, na Reserva Ecológica de Gurjaú, Pernambuco, através contagem por pontos.

(março de 2003) a $80 \pm 8$ (novembro de 2002) (Fig. 2). No período chuvoso (agosto, março eabril), também considerado não reprodutivo, as espécies de aves tendem a diminuir suas vocal izações, o que as torna menos evidentes. O oposto, também, foi observado nos meses de estiagem ou reprodutivo. Resultados semelhantes foram obtidos por Aleixo \& VIELLIARD (1995), que encontraram a menor média no mês de março ea maior no mês de setembro.

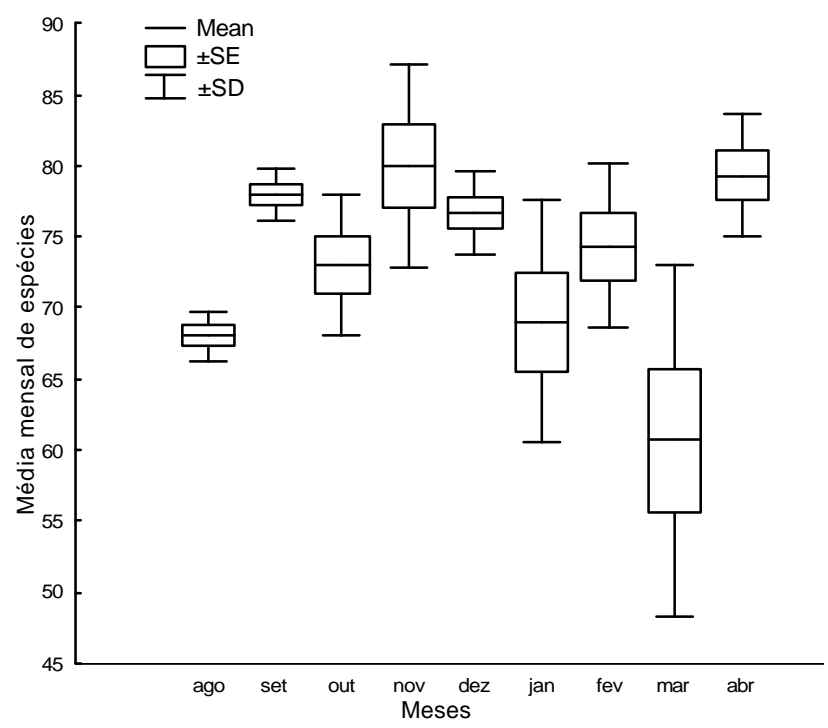

Figura 2. Variação da média mensal de espécies registradas por visita na Reserva Estadual de Gurjaú, Pernambuco, por meio da contagem por pontos.

O IPA encontrado por espécie variou entre 0,004 (um contato) em 23 espécies e 1,263 (341 contatos) apenas em Thraupis palmarum (Tab. I). A ordenação dos IPAs em ordem decrescente encontra-se na figura 3. 


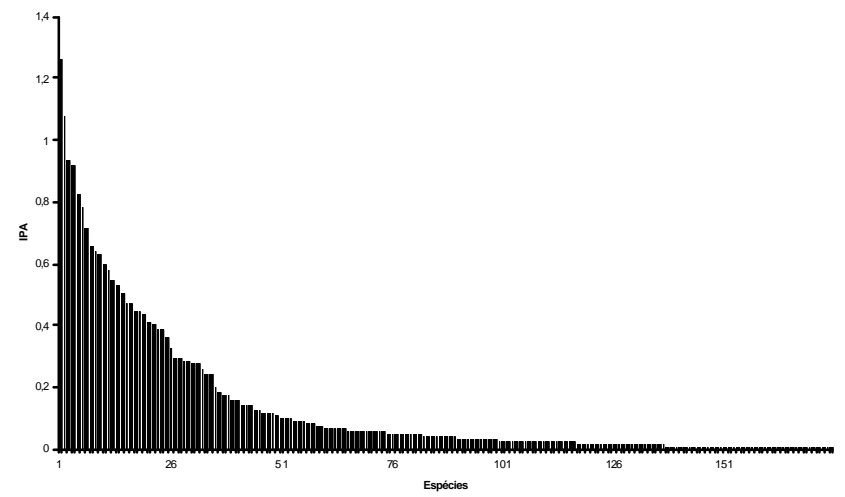

Figura 3. Ordenação decrescente dos Índices Pontuais de Abundância das espécies de aves registradas no levantamento quantitativo na Reserva Estadual de Gurjaú, Pernambuco.

O IPA mensal variou entre 14,0 e 28,9 (variação de 106\%) (Fig. 4). A variação encontrada por A teixo \& Vielliard (1995), com 202 amostras, foi de $65 \%(18,5$ a 30,6). Trabal hos realizados por VIeLLIARD \& SI LVA (1990) (110 amostras) obtiveram variação de $112 \%$ (12,6 e 26,7). O alto coeficiente de variação é decorrente da maior conspicuidade das manifestações sonoras, indicando o ciclo de abundância e atividade da avifauna considerada (Aleixo \& Vielliard 1995, Almeida et al. 1999).

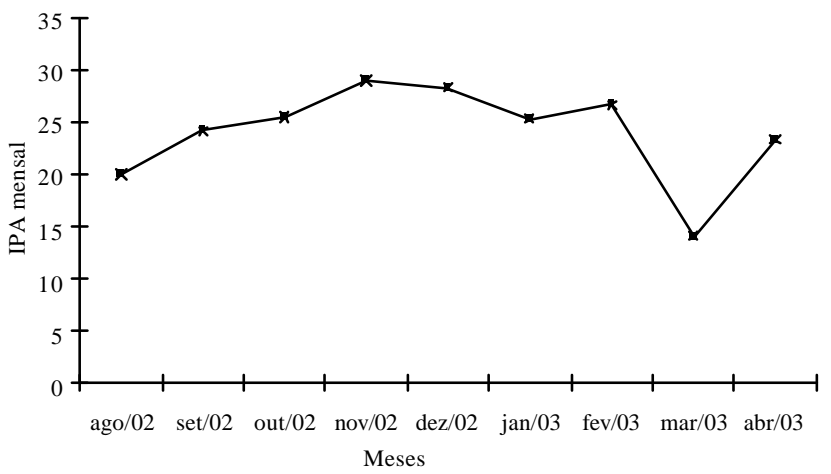

Figura 4. IPA médio mensal das espécies de aves catalogadas por meio do levantamento quantitativo na Reserva Ecológica de Gurjaú, Pernambuco.

O menor IPA mensal ocorreu no mês de março devido à diminuição das vocal izações das espécies de aves em decorrência de chuvas torrenciais ocorridas nesse mês. De acordo com ViellaARD \& Silva (1990) o clima é um fator que pode alterar de maneira considerável o grau de manifestações das espécies, explicando as alterações bruscas do IPA global. Os maiores valores de IPA mensal ocorreram entre os meses de setembro e feve reiro (Fig. 4). Neste período, considerado reprodutivo, há um aumento na abundância eo número deespécies registradas em decorrência da maior conspicuidade, discutida anteriormente. A diversidade da avifauna de Gurjaú foi de $\left(\mathrm{H}^{\prime}=4,25\right)$ ea eqüitabilidade ( $E=82,2 \%$ ). Os resultados de ambos os índices foram bastante altos. Em pesquisas realizadas por $M_{\text {ARSDEN }}$ et al. (2001) e Vielliard \& Si iva (1990), utilizando o método de contagem por pontos de escuta, também foram observadasaltas diversidades da avifauna $\left(\mathrm{H}^{\prime}=3,93\right.$ e $\left.\mathrm{H}^{\prime}=3,89\right)$ respectivamente.

Segundo M agurRan (1988) o Índice de Shannon-Wiener varia de 1,5 a 3,5, podendo raramente ultrapassar o val or de 4,5 e a eqüitabilidade varia entre 0 a $100 \%$, onde $100 \%$ indica espécies igual mente abundantes no ambiente. Os dados revelam alta diversidade de espécies em Gurjaú, as quais apresentam distribuição bastante equilibrada.

Trabal hos real izados através de metodologias qual itativas equantitativas associadas, contribuem para o conhecimento da composição avifaunística em fragmentos florestais, principalmente, aqueles localizados nos neotrópicos.

Observou-se a presença de grandes frugívoros, importantes na manutenção de fragmentos florestais: Crypturellus soui (Hermann, 1783), Trogon viridis Linnaeus, 1766, Trogon curucui Linnaeus, 1766, Pteroglossus aracari (Linnaeus, 1758) ePteroglossus inscriptus Swainson, 1822). A freqüência de ocorrência destas espécies foi de $18,5 \% ; 11,1 \% ; 29,6 \% ; 77,8 \%$ e $33,3 \%$, respectivamente (Tab. I), sen do três delas consideradas residentes para a área.

Dezesseis espécies apresentam alta sensibilidade a distúrbios ambientais de acordo com PARKER III et al. (1996), são elas: Micrastur gilvicollis (Vieillot, 1817); Aramides cajanea (Müller, 1776); Touit surda (Kuhl, 1820); Picumnus fulvescens Stager, 1961; Thamnophilus aethiops Sclater, 1858; Thamnomanes caesius (Temminck, 1820); Conopophaga melanops; Lepidocolaptes fuscus (Vieillot, 1818); Hemitriccus zosterops; Rhynchocyclus olivaceus (Temminck, 1820); Myiobius barbatus; Rhytipterna simplex (Linchtenstein, 1823); Pipra rubrocapilla Temminck, 1821; Chiroxiphia pareola; Schiffornis turdinus (Wied, 1831) e Curaeus forbesi (Sclater, 1886). Destas, doze são consideradas residentes, e oito tiveram a freqüência de ocorrência acima de $50 \%$, para as demais o percentual foi inferior a $26 \%$ (Tab. I).

Menciona-se a seguir as espécies endêmicas de Mata Atlântica, deacordo com StatTerfield et al.(1998) eas ameaçadas de extinção segundo critérios do M inistério do M elo Ambiente (2003).

Endêmicas: Touit surda (Kuhl, 1820); M elanotrochilus fuscus (Vieillot, 1817); Lepidocolaptesfuscus (Vieillot, 1818); Hylophilus poicilotis Temminck, 1822; Ramphocelus bresilius (Linnaeus, 1766).

Ameaçadas: Picumnus exilis Lichtenstein, 1823; Thamnophilus caerulescens Vieillot, 1816; Thamnophilus aethiops Sclater, 1858; Pyrigl ena leuconota (Spix, 1824); X enops minutus (Sparrman, 1788); Platyrinchus mystaceus Vieillot, 1818 e Schiffornis turdinus (Wied, 1831). 
Tabela I. Listagem das espécies de aves registradas para a Reserva Ecológica de Gurjaú, através de metodologia quali-quantitativa, com o número de contatos (N), a Freqüência de Ocorrência (FO), o Índice Pontual de Abundância (IPA) e o status: ST1 = residente (res), vagante (vag), ocasional (oc) e migratória (mig); ST2 = mata (M), borda (B), áreas abertas (A), açude (Aç) e para todas as áreas (T); ST3 = Sensibilidade a distúrbios, baixa (B), média (M) e alta (A).

\begin{tabular}{|c|c|c|c|c|c|c|c|}
\hline Espécies & $\mathrm{N}$ & $\mathrm{FO} \%$ & $\mathrm{~N}$ & IPA & ST1 & $\mathrm{ST} 2$ & ST3 \\
\hline \multicolumn{8}{|l|}{ Tinamidae } \\
\hline Crypturellus soui (Hermann, 1783) & 5 & 18,50 & 5 & 0,019 & vag & M & B \\
\hline Crypturellus parvirostris (Wagler, 1827) & 3 & 11,10 & 3 & 0,011 & oc & B & B \\
\hline Crypturellus tataupa (Temminck, 1815) & 3 & 11,10 & & - & vag & $A$ & B \\
\hline Nothura boraquira (Spix, 1825) & 2 & 7,41 & 2 & 0,007 & oc & A/B & M \\
\hline \multicolumn{8}{|l|}{ Podicipedidae } \\
\hline Tachybaptus dominicus (Linnaeus, 1766) & 5 & 18,50 & & - & vag & $A C ̧$ & M \\
\hline Podilymbus podiceps (Linnaeus, 1758) & 2 & 7,41 & 2 & 0,007 & vag & $\mathrm{AÇ}$ & M \\
\hline \multicolumn{8}{|l|}{ Ardeidae } \\
\hline Casmerodius albus (Linnaeus, 1758) & 5 & 18,50 & & - & oc & AÇ & B \\
\hline Egretta thula (Molina, 1782) & 1 & 3,70 & 9 & 0,033 & vag & $\mathrm{AÇ}$ & B \\
\hline Bulbucus ibis (Linnaeus, 1758) & 6 & 22,20 & 1 & 0,004 & res & AÇ & B \\
\hline Butorides striatus (Linnaeus, 1758) & 1 & 3,70 & 1 & 0,004 & vag & AÇ & B \\
\hline Tigrisoma lineatum (Boddaert, 1783) & 5 & 18,50 & 5 & 0,019 & vag & AÇ & M \\
\hline Ixobrychus exilis (Gmelin, 1789) & 2 & 7,41 & & - & vag & AÇ & M \\
\hline Botaurus pinnatus (Wagler, 1829) & 1 & 3,70 & 1 & 0,004 & vag & AÇ & M \\
\hline \multicolumn{8}{|l|}{ Cathartidae } \\
\hline Coragyps atratus (Bechstein, 1793) & 14 & 51,90 & 18 & 0,067 & res & $\mathrm{B} / \mathrm{A}$ & B \\
\hline Cathartes aura Linnaeus, 1758 & 15 & 55,60 & 19 & 0,070 & res & $\mathrm{T}$ & B \\
\hline Cathartes burrovianus Cassin, 1845 & 4 & 14,80 & & - & vag & $\mathrm{B} / \mathrm{A}$ & M \\
\hline \multicolumn{8}{|l|}{ Anatidae } \\
\hline Dendrocygna viduata (Linnaeus, 1766) & 10 & 37,00 & & - & oc & AÇ & B \\
\hline Amazonetta brasiliensis (Gmelin, 1789) & 5 & 18,50 & & - & vag & AÇ & B \\
\hline Nomonyx dominicus (Linnaeus, 1766) & 6 & 22,20 & & - & res & $A$ & M \\
\hline \multicolumn{8}{|l|}{ Accipitridae } \\
\hline Elanus leucurus (Vieillot, 1818) & 6 & 22,20 & & - & res & $A$ & B \\
\hline Gampsonyx swainsonii Vigors, 1825 & 1 & 3,70 & & - & vag & A & B \\
\hline Leptodon cayanensis (Latham, 1790) & 10 & 37,00 & 11 & 0,041 & res & M & M \\
\hline Buteo albicaudatus Vieillot, 1816 & 3 & 11,10 & & - & vag & $M / B$ & B \\
\hline Buteo brachyurus Vieillot, 1816 & 2 & 7,41 & 2 & 0,007 & vag & $M / B$ & M \\
\hline Asturina nitida (Latham, 1790) & 3 & 11,10 & 3 & 0,011 & vag & M & M \\
\hline Rupornis magnirostris (Gmelin, 1789) & 16 & 59,30 & 25 & 0,093 & res & $\mathrm{T}$ & B \\
\hline Buteogallus urubitinga (Gmelin, 1788) & 1 & 3,70 & 1 & 0,004 & vag & $M / B$ & M \\
\hline Spizaetus tyrannus (Wied, 1820) & 3 & 11,10 & 3 & 0,011 & vag & M & M \\
\hline \multicolumn{8}{|l|}{ Falconidae } \\
\hline Herpetotheres cachinnans Linnaeus, 1758 & 4 & 14,80 & 5 & 0,019 & vag & M & B \\
\hline M icrastur semitorquatus (Vieillot, 1817) & 1 & 3,70 & 1 & 0,004 & vag & M & M \\
\hline Micrastur gilvicollis (Vieillot, 1817) & 3 & 11,10 & 3 & 0,011 & vag & M & A \\
\hline Milvago chimachima (Vieillot, 1816) & 2 & 7,41 & 2 & 0,007 & oc & $\mathrm{B} / \mathrm{A}$ & B \\
\hline Caracara plancus (Miller, 1777) & 2 & 7,41 & 3 & 0,011 & oc & $\mathrm{T}$ & B \\
\hline \multicolumn{8}{|l|}{ Aramidae } \\
\hline Aramus guarauna (Linnaeus, 1766) & 2 & 7,41 & 2 & 0,007 & vag & $A C ̧$ & $\begin{array}{c}\text { B } \\
\text { ntinua }\end{array}$ \\
\hline
\end{tabular}


Tabela I. Continuação.

\begin{tabular}{|c|c|c|c|c|c|c|c|}
\hline Espécies & $\mathrm{N}$ & $\mathrm{FO} \%$ & $\mathrm{~N}$ & IPA & ST1 & ST2 & ST3 \\
\hline \multicolumn{8}{|l|}{ Rallidae } \\
\hline Aramides cajanea (Müller, 1776) & 3 & 11,10 & 3 & 0,011 & vag & $A C ̧ / B$ & A \\
\hline Porzana albicollis (Vieillot, 1819) & 1 & 3,70 & & & vag & $\mathrm{AÇ}$ & M \\
\hline Laterallus viridis (Müller, 1776) & 18 & 66,70 & 34 & 0,126 & res & $A C ̧$ & B \\
\hline Gallinula chloropus (Linnaeus, 1758) & 7 & 25,90 & & - & res & $A C ̧$ & B \\
\hline Porphyrula martinica (Linnaeus, 1766) & 25 & 92,60 & 76 & 0,281 & res & $A C ̧$ & B \\
\hline \multicolumn{8}{|l|}{ Jacanidae } \\
\hline Jacana jacana (Linnaeus, 1766) & 27 & 100,00 & 64 & 0,237 & res & $A C ̧$ & B \\
\hline \multicolumn{8}{|l|}{ Charadriidae } \\
\hline Vanellus chilensis (Molina, 1782) & 4 & 14,80 & 4 & 0,015 & oc & $\mathrm{A} / \mathrm{AÇ}$ & B \\
\hline \multicolumn{8}{|l|}{ Columbidae } \\
\hline Columbina passerina (Linnaeus, 1758) & 7 & 25,90 & & - & oc & $\mathrm{B} / \mathrm{A}$ & B \\
\hline Columbina minuta (Linnaeus, 1766) & 9 & 33,30 & 9 & 0,033 & res & $\mathrm{B} / \mathrm{A}$ & B \\
\hline Columbina talpacoti (Temminck, 1811) & 6 & 22,20 & 8 & 0,030 & res & $\mathrm{B} / \mathrm{A}$ & B \\
\hline Leptotila rufaxilla (Richard \& Bernard, 1792) & 27 & 100,00 & 79 & 0,293 & res & $M / B$ & M \\
\hline Geotrygon montana (Linnaeus, 1758) & 9 & 33,30 & 11 & 0,041 & res & $M / B$ & M \\
\hline \multicolumn{8}{|l|}{ Psittacidae } \\
\hline Aratinga leucophthalmus (Muller, 1776) & 2 & 7,41 & 1 & 0,004 & vag & M & B \\
\hline Aratinga cactorum (Kuhl, 1820) & 2 & 7,41 & 2 & 0,007 & vag & $M / B$ & M \\
\hline Forpus xanthopterygius (Spix, 1824) & 10 & 37,00 & 10 & 0,037 & res & $\mathrm{T}$ & B \\
\hline Touit surda (Kuhl, 1820) & 14 & 51,90 & 17 & 0,063 & res & $M / B$ & A \\
\hline Pionus maximiliani (Kuhl, 1820) & 23 & 85,20 & 37 & 0,137 & res & $M / B$ & M \\
\hline \multicolumn{8}{|l|}{ Cuculidae } \\
\hline Piaya cayana (Linnaeus, 1766) & 22 & 81,5 & 48 & 0,178 & res & $\mathrm{M} / \mathrm{B}$ & B \\
\hline Crotophaga ani Linnaeus, 1758 & 24 & 88,9 & 43 & 0,159 & res & $\mathrm{B} / \mathrm{A}$ & B \\
\hline Guira guira (Gmelin, 1788) & 10 & 37 & & - & res & A & B \\
\hline Tapera naevia (Linnaeus, 1766) & 2 & 7,41 & 2 & 0,007 & oc & $\mathrm{B} / \mathrm{A}$ & B \\
\hline \multicolumn{8}{|l|}{ Strigidae } \\
\hline Otus choliba (Vieillot, 1817) & 11 & 40,7 & & - & res & M & B \\
\hline Pulsatrix perspicillata (Latham, 1790) & 7 & 25,9 & & - & res & M & B \\
\hline Glaucidium brasilianum (Gmelin, 1788) & 1 & 3,7 & 1 & 0,004 & vag & $\mathrm{M} / \mathrm{B}$ & B \\
\hline \multicolumn{8}{|l|}{ Caprimulgidae } \\
\hline Nictidromus albicollis (Gmelin, 1789) & 1 & 3,7 & 1 & 0,004 & vag & A & B \\
\hline Hydropsalis brasiliana (Gmelin, 1798) & 11 & 40,7 & & - & res & A & B \\
\hline \multicolumn{8}{|l|}{ Apodidae } \\
\hline Tachornis squamata (Cassin, 1853) & 4 & 14,8 & 6 & 0,022 & oc & $\mathrm{B} / \mathrm{A}$ & B \\
\hline \multicolumn{8}{|l|}{ Trochilidae } \\
\hline Glaucis hirsuta (Gmelin, 1788) & 18 & 66,7 & 54 & 0,200 & res & $\mathrm{T}$ & B \\
\hline Phaethornis pretrei (Lesson \& Delattre, 1839) & 4 & 14,8 & 5 & 0,019 & vag & $M / B$ & B \\
\hline Phaethornis ruber (Linnaeus, 1758) & 27 & 100 & 88 & 0,326 & res & $\mathrm{M} / \mathrm{B}$ & M \\
\hline Eupetomena macroura (Gmelin, 1788) & 4 & 14,8 & 7 & 0,026 & vag & $\mathrm{T}$ & B \\
\hline Melanotrochilus fuscus (Vieillot, 1817) & 4 & 14,8 & & - & vag & A & M \\
\hline Anthracothorax nigricollis (Boddaert, 1783) & 9 & 33,3 & 13 & 0,048 & res & $\mathrm{M} / \mathrm{B}$ & B \\
\hline Chrysolampis mosquitus (Linnaeus, 1758) & 3 & 11,10 & 16 & 0,059 & mig & $\mathrm{M} / \mathrm{B}$ & $\mathrm{B}$ \\
\hline
\end{tabular}


Tabela I. Continuação.

\begin{tabular}{|c|c|c|c|c|c|c|c|}
\hline Espécies & $\overline{\mathrm{N}}$ & $\mathrm{FOO} \%$ & $\overline{\mathrm{N}}$ & IPA & "ST1 & $\overline{\mathrm{ST} 2}$ & 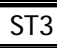 \\
\hline \multicolumn{8}{|l|}{ Trochilidae (continuação) } \\
\hline Chlorestes notatus (C. Reichenbach, 1795) & 15 & 55,60 & 26 & 0,096 & res & $\mathrm{T}$ & B \\
\hline Chlorostilbon aureoventris (d'Orbigny \& Lafresnaye, 1838) & 3 & 11,10 & 3 & 0,011 & vag & M/B & B \\
\hline Thalurania watertonii (Bourcier, 1847) & 7 & 25,90 & 12 & 0,044 & res & M/B & M \\
\hline Hylocharis cyanus (Vieillot, 1818) & 5 & 18,50 & 5 & 0,019 & vag & M & B \\
\hline Amazilia versicolor (Vieillot, 1818) & 1 & 3,70 & 1 & 0,004 & vag & $\mathrm{T}$ & B \\
\hline Amazilia fimbriata (Gmelin, 1788) & 2 & 7,41 & 2 & 0,007 & vag & $\mathrm{T}$ & B \\
\hline Amazilia leucogaster (Gmelin, 1788) & 2 & 7,41 & 2 & 0,007 & vag & $\mathrm{B} / \mathrm{A}$ & B \\
\hline Heliothryx aurita (Gmelin, 1788) & 4 & 14,80 & 4 & 0,015 & vag & $\mathrm{B} / \mathrm{A}$ & M \\
\hline Heliactin cornuta (Wied, 1821) & 1 & 3,70 & & - & mig & $M / B$ & M \\
\hline \multicolumn{8}{|l|}{ Trogonidae } \\
\hline Trogon viridis Linnaeus, 1766 & 3 & 11,10 & 3 & 0,011 & vag & & M \\
\hline Trogon curucui Linnaeus, 1766 & 8 & 29,60 & 8 & 0,030 & res & & M \\
\hline \multicolumn{8}{|l|}{ Alcedinidae } \\
\hline Ceryle torquata (Linnaeus, 1766) & 7 & 25,90 & & - & res & AÇ & B \\
\hline Chloroceryle amazona (Latham, 1790) & 2 & 7,41 & 2 & 0,007 & vag & AÇ & B \\
\hline Chloroceryle americana (Gmelin, 1788) & 11 & 40,70 & 16 & 0,059 & res & AÇ & B \\
\hline Chloroceryle aenea (Pallas, 1764) & 1 & 3,70 & 1 & 0,004 & vag & AÇ & M \\
\hline \multicolumn{8}{|l|}{ Galbulidae } \\
\hline Galbula ruficauda Curvier, 1816 & 26 & 96,30 & 77 & 0,285 & res & $\mathrm{T}$ & B \\
\hline \multicolumn{8}{|l|}{ Bucconidae } \\
\hline Nystalus maculatus (Gmelin, 1788) & 4 & 14,80 & 4 & 0,015 & oc & $\mathrm{T}$ & M \\
\hline \multicolumn{8}{|l|}{ Ramphastidae } \\
\hline Pteroglossus aracari (Linnaeus, 1758) & 21 & 77,80 & 29 & 0,107 & res & M/B & M \\
\hline Pteroglossus inscriptus Swainson, 1822 & 9 & 33,30 & 9 & 0,033 & res & M/B & M \\
\hline \multicolumn{8}{|l|}{ Picidae } \\
\hline Picumnus cirratus Temminck, 1825 & 5 & 18,50 & 8 & 0,030 & vag & M/B & B \\
\hline Picumnus exilis Lichtenstein, 1823 & 17 & 63,00 & 27 & 0,100 & res & M/B & M \\
\hline Picumnus fulvescens Stager, 1961 & 16 & 59,30 & 22 & 0,081 & res & M/B & $A$ \\
\hline Dryocopus lineatus (Bangs \& Penard, 1918) & 1 & 3,70 & 1 & 0,004 & vag & M & B \\
\hline Veniliornis passerinus (Linnaeus, 1766) & 7 & 25,90 & 8 & 0,030 & res & M/B & B \\
\hline Veniliornis affinis (Swainson, 1821) & 13 & 48,10 & 17 & 0,063 & res & M & M \\
\hline \multicolumn{8}{|l|}{ Thamnophilidae } \\
\hline Taraba major (Vieillot, 1816) & 12 & 44,40 & & - & res & M/B & B \\
\hline Thamnophilus doliatus (Linnaeus, 1764) & 2 & 7,41 & & - & vag & $\mathrm{B} / \mathrm{A}$ & B \\
\hline Thamnophilus palliatus (Lichtenstein, 1823) & 9 & 33,30 & 11 & 0,041 & res & M/B & B \\
\hline Thamnophilus caerulescens Vieillot, 1816 & 10 & 37,00 & 13 & 0,048 & res & M & B \\
\hline Thamnophilus aethiops Sclater, 1858 & 18 & 66,70 & 25 & 0,093 & res & M & $A$ \\
\hline Dysithamnus mentalis (Temminck, 1823) & 11 & 40,70 & 12 & 0,044 & res & M/B & M \\
\hline Thamnomanes caesius (Temminck, 1820) & 10 & 37,00 & 12 & 0,044 & res & M & A \\
\hline Myrmotherula axillaris Vieillot, 1817 & 25 & 92,60 & 80 & 0,296 & res & M/B & M \\
\hline Herpsilochmus rufimarginatus (Temminck, 1822) & 26 & 96,30 & 103 & 0,381 & res & M/B & M \\
\hline Herpsilochmus atricapillus Pelzeln, 1868 & 11 & 40,70 & 15 & 0,056 & res & M/B & M \\
\hline Formicivora grisea (Boddaert, 1783) & 24 & 88,90 & 111 & 0,411 & res & M/B & B \\
\hline
\end{tabular}


Tabela I. Continuação.

\begin{tabular}{|c|c|c|c|c|c|c|c|}
\hline Espécies & $\mathrm{N}$ & FO\% & $\mathrm{N}$ & IPA & ST1 & $\mathrm{ST} 2$ & ST3 \\
\hline \multicolumn{8}{|l|}{ Thamnophilidae (continuação) } \\
\hline Pyriglena leuconota (Spix, 1824) & 1 & 3,70 & & - & vag & M & M \\
\hline Myrmeciza ruficauda (Wied, 1831) & 7 & 25,90 & 11 & 0,041 & res & $M$ & M \\
\hline \multicolumn{8}{|l|}{ Conopophagidae } \\
\hline Conopophaga melanops (Wied, 1831) & 1 & 3,70 & & - & vag & M & $A$ \\
\hline Conopophaga lineata (Vieillot, 1818) & 1 & 3,70 & & - & vag & M & M \\
\hline \multicolumn{8}{|l|}{ Furnariidae } \\
\hline Furnarius leucopus Swainson, 1838 & 20 & 74,10 & & - & res & A & B \\
\hline Synallaxis frontalis Pelzeln, 1859 & 8 & 29,60 & 11 & 0,041 & res & $M / B$ & $\mathrm{~B}$ \\
\hline Poecilurus scutatus (Sclater, 1859) & 3 & 11,10 & 4 & 0,015 & vag & B & M \\
\hline Certhiaxis cinnamomea Gmelin, 1788 & 10 & 37,00 & 10 & 0,037 & res & $A C ̧$ & M \\
\hline Phacellodomus rufifrons (Wied, 1821) & 19 & 70,40 & 27 & 0,100 & res & $M / B$ & M \\
\hline Xenops minutus (Sparrman, 1788) & 8 & 29,60 & 13 & 0,048 & res & M & M \\
\hline Xenops rutilans Temminck, 1821 & 3 & 11,10 & 3 & 0,011 & vag & B & M \\
\hline \multicolumn{8}{|l|}{ Dendrocolaptidae } \\
\hline Sittasomus griseicapillus (Vieillot, 1818) & 26 & 96,30 & 108 & 0,400 & res & $M / B$ & M \\
\hline Xiphorhynchus picus (Gmelin, 1788) & 25 & 92,60 & 74 & 0,274 & res & $M / B$ & B \\
\hline Lepidocolaptes angustirostris (Vieillot, 1918) & 1 & 3,70 & 1 & 0,004 & vag & M & M \\
\hline Lepidocolaptes fuscus (Vieillot, 1818) & 14 & 51,90 & 17 & 0,063 & res & M & $A$ \\
\hline \multicolumn{8}{|l|}{ Tyrannidae } \\
\hline Zimmerius gracilipes (Sclater \& Salvin, 1867) & 25 & 92,60 & 119 & 0,441 & res & $M / B$ & M \\
\hline Camptostoma obsoletum (Temminck, 1824) & 17 & 63,00 & 34 & 0,126 & res & $M / B$ & B \\
\hline Phaeomyias murina (Spix, 1825) & 14 & 51,90 & & - & res & $M / B$ & B \\
\hline Myiopagis viridicata (Vieillot, 1817) & 14 & 51,90 & & - & res & $M / B$ & M \\
\hline M yiopagis gaimardii (d'Orbigny, 1839) & 7 & 25,90 & 15 & 0,056 & res & $M / B$ & M \\
\hline Elaenia flavogaster (Thunberg, 1822) & 27 & 100,00 & 251 & 0,930 & res & $M / B$ & B \\
\hline Elaenia spectabilis (Pelzeln, 1868) & 3 & 11,10 & 3 & 0,011 & mig & $\mathrm{B} / \mathrm{A}$ & B \\
\hline Elaenia mesoleuca (Cabanis \& Heine, 1859) & 2 & 7,41 & 2 & 0,007 & vag & $\mathrm{B} / \mathrm{A}$ & B \\
\hline Elaenia cristata (Pelzeln, 1868) & 1 & 3,70 & 1 & 0,004 & vag & $\mathrm{B} / \mathrm{A}$ & M \\
\hline Mionectes oleagineus (Lichtenstein, 1823) & 6 & 22,20 & 7 & 0,026 & res & $M / B$ & M \\
\hline Leptopogon amaurocephalus Tschudi, 1846 & 18 & 66,70 & 64 & 0,237 & res & $M / B$ & M \\
\hline Capsiempis flaveola (Lichtenstein, 1823) & 25 & 92,60 & 37 & 0,137 & res & $M / B$ & B \\
\hline Hemitriccus margaritaceiventer (Lafresnaye $\&$ d'Orbigny, 1837) & 13 & 48,10 & 13 & 0,048 & res & B & M \\
\hline Hemitriccus zosterops (Pelzeln, 1868) & 27 & 100,00 & 178 & 0,659 & res & $M / B$ & A \\
\hline Todirostrum cinereum (Linnaeus, 1766) & 27 & 100,00 & 136 & 0,504 & res & $\mathrm{T}$ & B \\
\hline Poecilotriccus fumifrons (Hartlaub, 1853) & 2 & 7,41 & 2 & 0,007 & vag & $M / B$ & $\mathrm{~B}$ \\
\hline Rhynchocyclus olivaceus (Temminck, 1820) & 15 & 55,60 & 21 & 0,078 & res & M & $A$ \\
\hline Tolmomyias sulphurescens (Spix, 1825) & 3 & 11,10 & 3 & 0,011 & vag & $M / B$ & M \\
\hline Tolmomyias poliocephalus (Taszanowski, 1884) & 7 & 25,90 & 24 & 0,089 & res & $M / B$ & M \\
\hline Tolmomyias flaviventris (Wied, 1831) & 27 & 100,00 & 157 & 0,581 & res & $\mathrm{T}$ & B \\
\hline Platyrinchus mystaceus Vieillot, 1818 & 1 & 3,70 & & - & vag & M & M \\
\hline M yiobius barbatus (Gmelin, 1789) & 1 & 3,70 & 1 & 0,004 & vag & M & $A$ \\
\hline Myiophobus fasciatus (Temminck, 1822) & 7 & 25,90 & 7 & 0,026 & res & $\mathrm{B} / \mathrm{A}$ & B \\
\hline Lathrotriccus euleri (Cabanis, 1868) & 3 & 11,10 & 3 & 0,011 & vag & $\mathrm{T}$ & M \\
\hline Cnemotriccus fuscatus (Wied, 1831) & 1 & 3,70 & 1 & 0,004 & vag & $\mathrm{T}$ & B \\
\hline
\end{tabular}


Tabela I. Continuação.

\begin{tabular}{|c|c|c|c|c|c|c|c|}
\hline Espécies & $\mathrm{N}$ & $\mathrm{FO} \%$ & $\mathrm{~N}$ & IPA & ST1 & ST2 & ST3 \\
\hline \multicolumn{8}{|l|}{ "Tyrannidae (Continuação) } \\
\hline Fluvicola nengeta (Vieillot, 1824) & 23 & 85,20 & 31 & 0,115 & res & $B / A$ & M \\
\hline Arundinicola leucocephala (Linnaeus, 1764) & 11 & 40,70 & & - & res & AÇ & M \\
\hline Machetornis rixosus (Vieillot, 1819) & 4 & 14,80 & 4 & 0,015 & vag & $\mathrm{B} / \mathrm{A}$ & B \\
\hline Rhytipterna simplex (Lichtenstein, 1823) & 5 & 18,50 & 5 & 0,019 & res & $M / B$ & $A$ \\
\hline M yiarchus ferox (Gmelin, 1789) & 11 & 40,70 & 16 & 0,059 & res & $\mathrm{T}$ & $\mathrm{B}$ \\
\hline M iyarchus tyrannulus (Müller, 1776) & 6 & 22,20 & 5 & 0,019 & res & $M / B$ & $\mathrm{~B}$ \\
\hline M yiarchus swainsoni (Berlepsch, 1883) & 5 & 18,50 & 5 & 0,019 & vag & $M / B$ & $\mathrm{~B}$ \\
\hline Myiarchus tuberculifer (Lafresnaye $\&$ d'Orbigny, 1837) & 25 & 92,60 & 74 & 0,274 & res & M & $\mathrm{B}$ \\
\hline Pitangus sulphuratus (Linnaeus, 1766) & 27 & 100,00 & 290 & 1,074 & res & $\mathrm{T}$ & $\mathrm{B}$ \\
\hline M egarhynchus pitangua (Linnaeus, 1766) & 19 & 70,40 & 32 & 0,119 & res & $\mathrm{T}$ & B \\
\hline M yiozetetes similis (Spix, 1825) & 27 & 100,00 & 171 & 0,633 & res & $\mathrm{T}$ & B \\
\hline M yiodynastes maculatus (Müller, 1776) & 1 & 3,70 & 1 & 0,004 & vag & $B / A$ & B \\
\hline Legatus leucophaius (Vieillot, 1818) & 18 & 66,70 & 119 & 0,441 & res & $M / B$ & B \\
\hline Empidonomus varius (Vieillot, 1818) & 1 & 3,70 & 1 & 0,004 & vag & B & B \\
\hline Tyrannus melancholicus Vieillot, 1819 & 25 & 92,60 & 126 & 0,467 & res & $\mathrm{T}$ & B \\
\hline Pachyramphus viridis (Vieillot, 1816) & 1 & 3,70 & 1 & 0,004 & vag & B & M \\
\hline Pachyramphus polychopterus (Vieillot, 1818) & 18 & 66,70 & 38 & 0,141 & res & $M / B$ & B \\
\hline Pachyramphus validus (Vieillot, 1816) & 3 & 11,10 & 3 & 0,011 & res & $M / B$ & M \\
\hline \multicolumn{8}{|l|}{ Pipridae } \\
\hline Pipra rubrocapilla Temminck, 1821 & 21 & 77,80 & 70 & 0,259 & res & M & $A$ \\
\hline Chiroxiphia pareola (Linnaeus, 1766) & 27 & 100,00 & 212 & 0,785 & res & $M / B$ & $A$ \\
\hline Manacus manacus (Linnaeus, 1766) & 26 & 96,30 & 117 & 0,433 & res & $M / B$ & B \\
\hline Neopelma pallescens (Lafresnaye, 1853) & 5 & 18,50 & 5 & 0,019 & vag & $M / B$ & $M$ \\
\hline Schiffornis turdinus (Wied, 1831) & 7 & 25,90 & 7 & 0,026 & res & M & $A$ \\
\hline \multicolumn{8}{|l|}{ Hyrundinidae } \\
\hline Tachycineta albiventer (Boddaert, 1783) & 26 & 96,30 & 48 & 0,178 & res & $A C ̧$ & B \\
\hline Progne chalybea (Gmelin, 1789) & 14 & 51,90 & & - & res & A & B \\
\hline Alopochelidon fucata (Temminck, 1822) & 1 & 3,70 & 1 & 0,004 & vag & A & M \\
\hline Stelgidopteryx ruficollis (Vieillot, 1817) & 20 & 74,10 & 47 & 0,174 & res & $B / A$ & B \\
\hline Hirundo rustica (Boddaert, 1783) & 3 & 11,10 & 3 & 0,011 & mig & A & B \\
\hline \multicolumn{8}{|l|}{ Troglodytidae } \\
\hline Donacobius atricapillus (Linnaeus, 1766) & 25 & 92,60 & 43 & 0,159 & res & $\mathrm{B} / \mathrm{AC}$ & M \\
\hline Thryothorus genibarbis Swainson, 1837 & 27 & 100,00 & 162 & 0,600 & res & $M / B$ & B \\
\hline Troglodytes musculus Naumann, 1823 & 27 & 100,00 & 126 & 0,467 & res & $\mathrm{T}$ & B \\
\hline \multicolumn{8}{|l|}{ Muscicapidae } \\
\hline Ramphocaenus melanurus Vieillot, 1819 & 24 & 88,90 & 142 & 0,526 & res & $M / B$ & B \\
\hline Polioptila plumbea (Gmelin, 1788) & 13 & 48,10 & 18 & 0,067 & res & $M / B$ & B \\
\hline Turdus rufiventris Vieillot, 1818 & 22 & 81,50 & 32 & 0,119 & res & $\mathrm{T}$ & B \\
\hline Turdus leucomelas Vieillot, 1818 & 27 & 100,00 & 192 & 0,711 & res & $M / B$ & B \\
\hline Turdus amaurochalinus Cabanis, 1851 & 1 & 3,70 & 1 & 0,004 & mig & M & B \\
\hline Turdus fumigatus Lichtenstein, 1823 & 2 & 7,41 & 2 & 0,007 & vag & M & M \\
\hline \multicolumn{8}{|l|}{ Vireonidae } \\
\hline Cyclarhis gujanensis (Gmelin, 1789) & 27 & 100,00 & 147 & 0,544 & res & $M / B$ & B \\
\hline Vireo chivi (Vieillot, 1817) & 27 & 100,00 & 247 & 0,915 & res & $M / B$ & B \\
\hline Hylophilus amaurocephalus (Nordmann, 1835) & 1 & 3,70 & 1 & 0,004 & vag & M & M \\
\hline
\end{tabular}


Tabela I. Continuação.

\begin{tabular}{|c|c|c|c|c|c|c|c|}
\hline Espécies & $\mathrm{N}$ & $\mathrm{FO} \%$ & $\mathrm{~N}$ & IPA & ST1 & ST2 & ST3 \\
\hline \multicolumn{8}{|l|}{ Emberizidae } \\
\hline Basileuterus flaveolus (Baird, 1865) & 15 & 55,60 & 15 & 0,056 & res & $\mathrm{M} / \mathrm{B}$ & M \\
\hline Basileuterus culicivorus (Lichtenstein, 1830) & 9 & 33,30 & & - & res & $\mathrm{M} / \mathrm{B}$ & M \\
\hline Coereba flaveola (Linnaeus, 1758) & 26 & 96,30 & 223 & 0,826 & res & $\mathrm{T}$ & B \\
\hline Cissopis leveriana (Gmelin, 1788) & 2 & 7,41 & & - & vag & $\mathrm{M} / \mathrm{B}$ & B \\
\hline Thlypopsis sordida (Lafresnaye \& d'Orbigny, 1837) & 1 & 3,70 & 1 & 0,004 & vag & $B / A$ & B \\
\hline Nemosia pileata (Boddaert, 1783) & 8 & 29,60 & 9 & 0,033 & res & $\mathrm{M} / \mathrm{B}$ & B \\
\hline Tachyphonus cristatus (Linnaeus, 1766) & 11 & 40,70 & 16 & 0,059 & res & $\mathrm{M} / \mathrm{B}$ & M \\
\hline Tachyphonus rufus (Boddaert, 1783) & 7 & 25,90 & 7 & 0,026 & res & $\mathrm{B} / \mathrm{A}$ & B \\
\hline Ramphocelus bresilius (Linnaeus, 1766) & 1 & 3,70 & & - & OC & $B / A$ & B \\
\hline Thraupis sayaca (Linnaeus, 1766) & 7 & 25,90 & 10 & 0,037 & res & $\mathrm{T}$ & B \\
\hline Thraupis palmarum (Wied, 1821) & 27 & 100,00 & 341 & 1,263 & res & $\mathrm{T}$ & B \\
\hline Euphonia chlorotica (Linnaeus, 1776) & 8 & 29,60 & 15 & 0,056 & res & $\mathrm{T}$ & B \\
\hline Euphonia violacea (Linnaeus, 1758) & 24 & 88,90 & 96 & 0,356 & res & $\mathrm{T}$ & $\mathrm{B}$ \\
\hline Tangara fastuosa (Lesson, 1831) & 14 & 51,90 & & - & res & $\mathrm{M} / \mathrm{B}$ & M \\
\hline Tangara cyanocephala (Müller, 1766) & 4 & 14,80 & & - & vag & $M / B$ & M \\
\hline Tangara cayana (Linnaeus, 1766) & 21 & 77,80 & 46 & 0,170 & res & $\mathrm{M} / \mathrm{B}$ & M \\
\hline Tangara velia (Wied, 1830) & 6 & 22,20 & & - & res & $M / B$ & M \\
\hline Dacnis cayana (Linnaeus, 1766) & 14 & 51,90 & 19 & 0,070 & res & $\mathrm{M} / \mathrm{B}$ & B \\
\hline Cyanerpes cyaneus (Linnaeus, 1766) & 7 & 25,90 & 13 & 0,048 & res & $\mathrm{M} / \mathrm{B}$ & B \\
\hline Volatinia jacarina (Linnaeus, 1766) & 6 & 22,20 & 8 & 0,030 & res & $B / A$ & B \\
\hline Sporophila lineola (Linnaeus, 1758) & 2 & 7,41 & & - & oc & A & B \\
\hline Sporophila nigricollis (Vieillot, 1823) & 1 & 3,70 & 1 & 0,004 & OC & A & B \\
\hline Sporophilla leucoptera (Vieillot, 1817) & 2 & 7,41 & 2 & 0,007 & OC & $\mathrm{B} / \mathrm{A}$ & $\mathrm{B}$ \\
\hline Sporophila bouvreuil (Sclater, 1864) & 1 & 3,70 & & - & OC & A & $M$ \\
\hline Tiaris fuliginosa (Wied, 1831) & 7 & 25,90 & & - & res & M & B \\
\hline Arremon taciturnus (Hermann, 1783) & 26 & 96,30 & 103 & 0,381 & res & $\mathrm{M} / \mathrm{B}$ & M \\
\hline Paroaria dominicana (Linnaeus, 1758) & 1 & 3,70 & & - & oc & $A$ & B \\
\hline Saltator maximus (Müller, 1776) & 27 & 100,00 & 173 & 0,641 & res & $\mathrm{M} / \mathrm{B}$ & B \\
\hline Cacicus solitarius (Vieillot, 1816) & 6 & 22,20 & & - & res & $M / B$ & B \\
\hline Icterus cayanensis (Linnaeus, 1766) & 7 & 25,90 & 7 & 0,026 & res & $\mathrm{T}$ & M \\
\hline Icterus jamacaii (Gmelin, 1788) & 2 & 7,41 & 2 & 0,007 & vag & $M / B$ & B \\
\hline Sturnella superciliaris (Bonaparte, 1850) & 2 & 7,41 & & - & OC & A & $\mathrm{B}$ \\
\hline Curaeus forbesi (Sclater, 1886) & 7 & 25,90 & 7 & 0,026 & res & $M / B$ & A \\
\hline Molothrus bonariensis (Gmelin, 1789) & 2 & 7,41 & & - & OC & A & $\mathrm{B}$ \\
\hline \multicolumn{8}{|l|}{ Passeridae } \\
\hline Passer domesticus (Linnaeus, 1758) & 13 & 48,10 & 15 & 0,056 & res & $A$ & B \\
\hline \multicolumn{8}{|l|}{ Estrildidae } \\
\hline Estrilda astrild (Vieillot, 1805) & 3 & 11,10 & 3 & 0,011 & $\mathrm{OC}$ & $A$ & - \\
\hline
\end{tabular}

Endêmicas e Ameaçadas: Thalurania watertonii (Boucier, 1847); M yrmeciza ruficauda (Wied, 1831); Conopophaga melanops (Wied, 1831); Conopophaga lineata (Vieillot, 1818); Tangara fastuosa (Lesson, 1831); T angara cyanocephala (Müller, 1766) e Curaeus forbesi.
Além destas, destaca-se Picumnus fulvescens Stager, 1961 espécie en dêmica dos Estados de Alagoas e Pernambuco.

Os levantamentos quali-quantitativos revelaram a presença de espécies dependentes de interior de mata (apesar da baixa abundância), daquel as consi deradas sen síveis à fragmen- 
Tabela II. Dados comparativos de censos realizados através do método de contagem por pontos de escuta, nos Estados de São Paulo, Espírito Santo e Pernambuco.

\begin{tabular}{|c|c|c|c|c|c|c|}
\hline Autores & Estado & Área (ha) & Contatos/amostra & № de contatos & $\mathrm{N} \cong$ de amostras & № de espécies \\
\hline Esta pesquisa & Pernambuco & $1.077,10$ & 24,00 & 6.470 & 270 & 175 \\
\hline Marsden et al. 2001 & Espírito Santo & 46,05 & 1,84 & 1.002 & 546 & 111 \\
\hline Almeida et al. 1999, frag1 & São Paulo & 37,00 & 7,50 & 829 & 110 & 69 \\
\hline Almeida et al. 1999, frag2 & São Paulo & 27,00 & 10,10 & 1.107 & 110 & 75 \\
\hline Aleixo \& Vielliard 1995 & São Paulo & 251,00 & 23,20 & 4.706 & 202 & 82 \\
\hline Vielliard \& Silva 1990 & São Paulo & - & 19,90 & 2.194 & 110 & 111 \\
\hline
\end{tabular}

tação, de frugívoros de grande porte e de espécies en dêmicase ameaçadas de extinção e de outras que utilizam a área a procura de recursos al imentares ou de abrigo. Os dados obtidos demonstram a importância de Gurjaú para manutenção dessas aves, sendo sua conservação de extrema importância para estas populações.

\section{A GRA DECIMENTOS}

Agradecemosà Coordenação deAperfeiçoamento de Pessoal de Nível Superior (CAPES), à Fundação Apolônio Sales de Apoio e Desenvolvimento à Pesquisa (FADURPE), ao Departamento de Estradas e Rodagens (DER), à Companhia Pernambucana de Meio Ambiente (CPRH) e à Companhia Pernambucana de Abastecimento de Água (COMPESA), pelo suporte dado no decorrer desta pesquisa.

\section{REFERÊN CIAS BIBLIOGRÁ FICAS}

Aleixo, A. 1999. Effects of selectivelogging on a bird community in the Brazilian Atlantic Forest. The Condor, Camarillo, 101: 537-548.

Aleixo, A. \& J.M.E. Vielliard 1995. Composição e dinâmica da avifauna da M ata de Santa Genebra, Campinas, São Paulo, Brasil. Revista Brasil ei ra deZoologia, Curitiba, 12 (3): 493511.

Almeida, M.E. de C.; J.M.E. Vielliard \& M.M. Dias. 1999. Composição da avifauna em duas matas ciliares na bacia do rio Jacaré-Pepira, São Paulo, Brasil. Revi sta Brasilei ra de Zoologia, Curitiba, 16 (4): 1087-1098.

Anjos, L. Dos. 1998. Conseqüências biológicas da fragmentação no norte do Paraná. IPEF, Curitiba, 12 (32): 87-94.

. 2001. Bird communities in five Atlantic Forest fragments in Southern Brazil. Ornitol ogia Neotropical, Montreal, 12: 11-27.

. 2002. Forest bird communities in Tibagi River Hydrographic Basin, Southern Brazil. Ecotropica, Bonn, 8: 67-79.

Anjos, L. dos \&. R. Boçon. 1999. Bird communities in natural forest patches in southern Brazil. The Wilson Bulletin, Lawrence, 111 (3): 397-414.

Askins, R.A.; M.J. Philbrick \& D.S. Sugeno. 1987. Relationship between the regional abundance of forest and the composi- tion of forest bird communities. Biological Conservation, Davis, 39: 129-152.

AzeVEdo-Júnior, S.M.DE. 1990. A Estação Ecológica do Tapacurá e suas aves. Anais do Encontro Nacional deAnilhadores de Aves (ENAV), Recife, 4: 92-99.

BierregaArd J R., R.O. \& T.E. Lovejoy. 1989. Effects of forest fragmentation on Amazonian understory bird communities. Acta Amazônica, Manaus, 19: 215-241.

Bierregaard JR., R.O.; T.E. Lovejoy; V. Kapos; A.A. dos Santos \& R.W. Hutchings. 1992. The biological dynamics of tropical rainforest fragments: a prospectivecomparison of fragments and continuous forest. BioScience, Washington, 42 (11): 859-866.

Blondel, J.; C. Ferry \& B. Frochot. 1970. La méthode des indices ponctuels d'abondance (I.P.A.) ou des relevés d'avifaune par "stations d'écoute". Alauda, Paris, 38: 55-71.

Brooks, T.M.; R.A. M ItTermeier; C.G. M Ittermeier; G.A.B. da Fonseca; A.B. Rylands; W.R. Konstant; P. Flick; J. Pilgrim; S. OldFIELD; G. M ANGIN \& C. HILton-TAYLoR. 2002. Habitat loss and extinction in the hotspots of biodiversity. Conservation Biology, Montpelier, 16 (4): 909-920.

FIDEM. 1987. Região M etropolitana do Recife: Reservas Ecológicas. Recife, Governo do Estado de Pernambuco, Secretaria de Planejamento do Estado de Pernambuco, Fundação de Desenvolvimento da Região M etropolitana do Recife, I+108p.

Gimenes, M.R. \& L. dos Anjos. 2000. Distribuição espacial de aves em um fragmento florestal do Campus da Universidade Estadual de Londrina, Norte do Paraná, Brasil. Revista Brasileira deZoologia, Curitiba, 17 (1): 263-271.

KRüguel, M.M. \& L. dos Anjos. 2000. Bird communities in Forest remnants in the city of Maringá, Paraná state, southern Brazil. Ornitologia Neotropical, Montreal, 11: 315-330.

Lyra-Neves, R.M. De; W.R. Telino-Júnior; R.C. Rodrigues \& M. da C.N. B отецно. 2000. Caracterização e avaliação da população avifaunística da APA de Guadal upe. Disponível em: http://www.cprh.pe.gov.br/sec-unidconserv/frme-secundunid.html, acesso em: 01 de agosto de 2003.

MagurRan, A.E. 1988. Ecological diversity and its measurement. London, Croom Helm, 179p.

M ARSDEN, S.J.; M. W hifFin \& M. Galettı. 2001. Bird diversity and 
abundance in forest fragments and Eucalyptus plantations around an Atlantic forest reserve, Brazil. Biodiversity and Conservation, Dordrecht, 10: 737-751.

M ARINI, M.Â. 2000. Efeitos da fragmentação florestal sobre as aves em Minas Gerais, p. 41-54. In: M.A. dos Santos-Alves; J.M.C. da Silva; M. van Sluys; H. de G. Bergallo \& C.F.D. da Rосна (Orgs). A ornitologia no Brasil: pesquisa atual e perspectivas. Rio deJaneiro, Editora UERJ, 352p.

Ministério do M eo Ambiente. 2003. Lista nacional das espécies da fauna brasil eira ameaçadas de extinção. Disponível em: http://www.mma.gov.br/port/sbf/fauna/lista.html, acesso em: 01 de agosto de 2003.

Myers, N.; R.A. Mittermeier; C.G. Mittermeier; G.A.B. da FonsecA \& J. KENT. 2000. Biodiversity hotspots for conservation priorities. Nature, London, 403: 853-858.

Parker III, T.A.; D.F. Stotz \& J.W. FitzPatrick. 1996. Ecological and distributional databases, p. 113-436. In: D.F. Stoтz; J.W. FitzPATRICK; T.A. PARKer III \& D.K. M OsKovits (Eds). Neotropical birds: ecology and conservation. Chicago, University of Chicago Press, XI+700p.

Primack, R.B. \& E. Rodrigues. 2001. Biologia da Con servação. Londrina, E. Rodrigues, 328p.

Ranta, P.; T. Blom; J. Niemelä; E. Joensuu \& M. Siitonen. 1998. The fragmented Atlantic Rain Forest of Brazil: size, shape and distribution of forest fragments. Biodiversity and Conservation, Dordrecht, 7: 385-403.

Silla, J.M.C. da \& M. Tabarelli. 2000. Tree species impoverishment and the future flora of theAtlantic Forest of northeast
Brazil. Nature, London, 404: 72-74.

Statterfield, A.J.; M.J. Crosby ; A.J. Long \& D.C. W ege. 1998. Endemic bird areas of the world. Cambridge, BirdLife International, $846 \mathrm{p}$.

Stotz D.F.; J.W. FitzPatrick; T.A. PARKer III \& D.K. M oskovits. 1996. (Eds). Neotropical birds: ecology and conservation. Chicago, University of Chicago Press, XI+700p.

Stouffer, P.C. \& R.O. BerregaARd JR. 1995a. Effects of forest fragmentation on understory hummingbirdsin Amazonian Brazil. Conservation Biology, Montpelier, 9 (5): 1085-1094. . 1995b. Use of Amazonian forest fragments by understory insectivorous birds. Ecology, Durham, 76 (8): 24292445.

VIELLIARD, J.M.E. 2000. Bird community as an indicator of biodiversity: results from quantitative surveys in Brazil. Anais da Academia Brasil ei ra de Ciências, Rio de Janeiro, 72 (3): 323-330.

Vielliard, J.M.E. \& W.R. Silva. 1990. Nova metodologia de levantamento quantitative da avifauna e primeiros resultados do interior do Estado de São Paulo, Brasil. In: An ais do IV Encontro Nacional deAnilhadoresdeAves, Recife, p. 117-151.

WEGE, D.C. \& A.J. Long. 1995. Key areas for threatened birds in the neotropics. BirdLife Conservation Series, Cambridge, 5: 75-80.

WILLIS, E.O. \& Y. O NIKI. 1992. Losses of São Paulo birdsareworse in the interior than in Atlantic forests. Ciência eCultura, Campinas, 44 (5): 326-328.

Recebido em 04.XI.2003; aceito em 27.VII.2004.

O arquivo disponível sofreu correções conforme ERRATA publicada no Volume22 Número 1 da revista. 